\title{
THIN CIRCULAR PLATE UNIFORMLY LOADED OVER A CONCENTRIC ELLIPTIC PATH AND SUPPORTED ON COLUMNS
}

\author{
W. A. BASSALI \\ Departinent of Mathematics \\ Faculty of Science \\ Kuwait University \\ Kuwait, P.o. Box 5969
}

(Received Apri1 29, 1985)

\begin{abstract}
Within the limitations of the classical thin plate theory expressions are obtained for the small deflections of a thin isotropic circular plate uniformly loaded over a concentric ellipse and supported by four columns at the vertices of a rectangle whose sides are parallel to the axes of the ellipse. Formulae are given for the monents and shears at the centre of the plate and on the edge. Limiting cases are investigated.
\end{abstract}

KEY WORDS AND PHRASES. Deflections of circular plates, four point supports, free boundary, uniform loading on a concentric ellipse

1980 AMS SUBJECT CLASSIFICATION CODE. 73N.

1. INTRODUCTION.

The technological importance of thin elastic plates is sufficiently well established to require no elaboration. Thin slabs of material are structures which are widely used in engineering work and their transverse flexure has been extensively studied by many authors both theoretically and experimentally when the boundary of the slab is clamped, simply supported or free. Support of circular discs at a discrete number of points is of interest to the designer of reflecting surfaces and receivers, particularly when the surfaces are parts of astronomical and aeronautical structures. The deflection surface of a thin circular plate subjected to a symmetrical loading and supported by equally spaced point columns along the periphery of the plate has been considered by Nadai [1] whose results are quoted by Timoshenko and Woinowsky-Krieger [2]. When the boundary of the circular plate is free and the plate is supported at interior points and acted upon by two types of normal loadings distributed over an eccentric circular patch the solutions have been obtained by the author [3,4], using the complex variable approach of Muskhelishvili, references to previous work are given at the ends of these papers. Thin circular plates on multipoint supports have also been discussed by Yu and Pan [5], Leissa and Wel1s [6], Kirstein, Pell, Woolley and Davis [7], Kirstein and Wooley [8,9], Vaughn [10], Chantaramungkorn, Karasudhi and Lee [11] and Williams and Brinson [12]. There is good asreement between the theoretical results of [3] and the experimental results from the 
tests repirted in [7]. Most of the results in [5-10,12] are special cases of [3]. In a sieries if papers [13-15] complex variable methods were applied to study the fending of an elastically restrained circular plate subject to uniform, linearly varying and partbolic loadings over a concentric ellipse. Frishbeir and Lucht [16] used the //L// method if complex potentials to derive the solution for a clamped circular plate which is transversely and unifuring loaded over the area of a polygon. In this paper, expressions are obtained for the deflection at any point of a thin circular plate which i; uniformly loaded over a concentric elliptic patch and supported by four equal concentrated forces locited at the corners of a rectangle whose sides are parallel to the axes of the ellipse. Formulae are given for the boundary and central values of the monents and shears. The limiting cases in which the radius of the plate $+\infty$, the eccenlricity of the ellipse $\rightarrow$ or its minor axis, 0 are investigated.

\section{BASIC EQUATIONS AND BOUNDARY CONDITIONS.}

Let $C$ denote the boundary of a thin circular plate of centre 0 anu radius c. If $2 \mathrm{~h}$ is the constant thickness of the plate, then its flexural rigidity $\mathrm{D}$ is given by [2]

$$
D=2 E h^{3} / 3\left(1-v^{2}\right)
$$

where $E$ is the modulus of elasticity and $\nu$ is Poisson's ratio for the material of the plate. The mid-plane of the plate is chosen as the plane $Z=0$ of a rectangular Cartesian frame $O(x, y, z)$ and the notation used is that of [2]. According to the classical small bending theory of thin plates the deflection $w$ of the midplane in the downward direction $O Z$ at any point $z=x+i y=r e^{i \cdot j}$ satisfies the biharmonic equation

$$
D v^{4} w=p(z, \bar{z}),
$$

where

$$
\nabla^{2}=\frac{\partial^{L}}{\partial x^{2}}+\frac{\partial^{2}}{\partial y^{2}}=4 \frac{\partial^{2}}{\partial z \partial \bar{z}}=\frac{\partial^{2}}{\partial r^{2}}+\frac{1}{r} \frac{\partial}{\partial r}+\frac{1}{r^{2}} \frac{\partial^{2}}{\partial \theta^{2}},
$$

and $p(z, \bar{z})$ is the normal load intensity at the point $z$. The general solution of (2.2) may be written as

$$
w=\bar{z} j(z)+\overline{z i c}(\bar{z})+w(z)+\bar{w}(\bar{z})+w(z, \bar{z}),
$$

where. $(z), w(z)$ are functions of $z$ which are regular in the region occupied by the plate and $W$ is a particular integral of (2.2). The moments and shears at any point $(r, \forall)$ of the mid-piane of the plate are given by [2]

$$
\begin{aligned}
& M_{r}=-D\left(d^{2}+v r^{-1} d+v r^{-2} d^{2}\right) w=-D\left[u \nabla^{2}+(1-v) d^{2}\right] w, \\
& M_{,}=-D\left(v d^{2}+r^{-1} d+r^{-2} d^{\prime 2}\right) w=-D\left[\nabla^{2}+(v-1) d^{2}\right] w, \\
& M_{r}=(1-v) D r^{-1}\left(d-r^{-1}\right) d^{\prime} w, \\
& Q_{r}=-D d\left(.^{2} w\right), \quad Q_{v}=-D r^{-1} d^{\prime}\left(v^{2} w\right),
\end{aligned}
$$


where $a=j /, r, d^{\prime}=\partial / d \cdot$. In terns of tue complex potentials $\ldots(z), \omega(z)$ and particular inlegral $W(z, \bar{z})$ we have $[3, p .730]$

$$
\begin{aligned}
& M_{r}+M_{i j}=-4(1+) D\left[2 \operatorname{Re} \delta^{\prime}+\partial{ }^{2} W / \partial z \partial \bar{z}\right], \\
& M_{r}-M_{U}+2 i M_{r \cdot j}=-4(1-v) D\left[z \Delta \partial^{\prime \prime}+z^{2}\left(\omega^{\prime \prime}+W^{\prime \prime}\right) / r^{2}\right], \\
& Q_{r}-i Q_{\theta}=-8 D z\left["+\partial^{3} W / \partial z^{2} \partial \bar{z}\right] / r,
\end{aligned}
$$

where accents denote differentiation with respect to $z$.

The conditions for the circular edge $C$ to be free are [2]

$$
\left(M_{r}\right)_{r=c}=0,\left(V_{r}\right)_{r=c}=\left(Q_{r}-\frac{1}{r} \frac{\left.\partial{ }_{r}\right)}{\partial}\right)_{r=c}=0 \text {. }
$$

Substitution trom $(2.5 a, c)$ and $(2.6)$ in $(2.9)$ leads to

$$
\left[f_{r}\left(d, d^{\prime}\right) w\right]_{r=c}=0,\left[F_{r}\left(d, d^{\prime}\right) w\right]_{r=c}=0
$$

where

$$
\begin{aligned}
& f_{r}\left(d, d^{\prime}\right)=d^{2}+\nu r^{-1} d+v r^{-2} d^{\prime 2}, \\
& F_{r}\left(d, d^{\prime}\right)=d^{3}+r^{-1} d^{2}-r^{-2} d+(2-v) r^{-1} d d^{\prime 2}+(\nu-3) r^{-3} d^{\prime 2} .
\end{aligned}
$$

From (2.Ja) and the first equation of (2.9) we see that

$$
v\left(v^{2} w\right)_{r=c}=(v-1)\left(d^{2} w\right)_{r=c}, v\left[d^{\prime}\left(\nabla^{2} w\right)\right]_{r=c}=(v-1)\left[d^{\prime} d^{2} w\right]_{r=c}
$$

Equation (2.5b) and the second equation of (2.6) then give

$$
\left(N_{, j}\right)_{r=c}=\frac{1-v^{2}}{v} \nu\left[d^{2} w\right]_{r=c},\left(Q_{\theta}\right)_{r=c}=\frac{1-v}{v} \cdot \frac{D}{c}\left[d^{\prime} d^{2} w\right]_{r=c} \text {, }
$$

from which we deduce that

$$
(1+v)^{c}\left(Q_{\nu}\right)_{r=c}=\left(d^{\prime} M_{\vartheta}\right)_{r=c} .
$$

This relation and the second equation of (2.9) serve to determine the periphery values of the shears in terms of the moment values.

\section{STATEMENT OF THE PROBLEM.}

The problem to be solved consists of determining the deflection surface of a thin circular plate of centre 0 and radius $c$ subjected to the following conditions:

(1) The boundary $C$ of the plate is fret.

(2) The total normal load $L=\pi p_{0} a b$

is uniformly distributed over the area of the ellipse

$$
x^{2} / a^{2}+y^{2} / b^{2}=1(0 \leq b \leq a \leq c) \text {. }
$$


(3) The plate is supported by four equal concentrated forces, each of magnitude $\mathrm{L} / 4$, and located at the points $\mathrm{P}_{\lambda}\left(\mathrm{z}_{\lambda}=\mathrm{se}{ }^{\mathrm{i} \gamma}, \lambda=1,2,3,4\right)$, where $0 \leq \mathrm{s}=\mathrm{c}$, $r_{1}=r, r_{2}=\pi-\gamma, r_{3}=\gamma-\pi, r_{4}=-\gamma, 0 \leq \gamma \leq \pi / 2$. The four points of support lie in the loaded or unloaded region according as $s^{2} \cos ^{2} \gamma / a^{2}+s^{2} \sin ^{2} \gamma / b^{2}$ is $1 \in s s$ or greater than 1. For $\gamma=0$ we have two supports at $( \pm s, 0)$ while for $\gamma=\pi / 2$ we have two supports at $(0, \pm s)$. See Figure 1. Symmetry with respect to both axes show that it is sufficient to find the deflection $w$ at any point $z$ in the positive quadrant. Deflections, moments and shears at the four points $\pm z, \pm \bar{z}$ are the same.

\section{METHOD AND SOLUTION.}

Let $\Gamma$ denote the boundary of the ellipse $(3.4 \mathrm{~b})$ and 1 et the indices 1 and 2 refer to the loaded region inside $\Gamma$ and the unloaded region between $\Gamma$ and $C$, respectively. The particular integrals $W_{1}$ and $W_{2}$ of (2.2) corresponding to the uniform intensities of normal loading $\mathrm{p}_{1}=\mathrm{p}_{0}$ and $\mathrm{p}_{2}=0$ may be taken as

$$
\mathrm{W}_{1}(\mathrm{z}, \overline{\mathrm{z}})=\mathrm{p}_{0} \mathrm{z}^{2-2} / 64 \mathrm{D}, \quad \mathrm{W}_{2}(\mathrm{z}, \overline{\mathrm{z}})=0 \text {. }
$$

The continuity requirements for the deflections, slopes, moments and shears at any point on $\Gamma$ lead to

$$
[w]_{1}^{2}=[\partial w / \partial z]_{1}^{2}=\left[\partial^{2} w / \partial z \partial \bar{z}\right]_{1}^{2}=\left[\partial^{3} w / \partial z^{2} \partial \bar{z}\right]_{1}^{2}=0
$$

along $\Gamma^{\prime}$. It was proved [13, p.105] that these transition conditions along $\Gamma$ are satisfied by

$[\operatorname{sic}(z)]_{1}^{2}=\frac{L}{96 \pi D}\left[\frac{d^{2} z^{3}}{a b f^{2}}-2\left(2+\frac{z^{2}}{f^{2}}\right) z+6 z \ln \frac{z+z}{a+b}\right]$,

$[\omega(z)]_{1}^{2}=-\frac{L}{96 \pi D}\left[\left(\frac{2 a b}{f^{4}}+\frac{1}{4 a b}\right) z^{4}-\frac{3}{2} a b\left(1+\frac{4 z^{2}}{f}\right)+d^{2}\left\{\frac{z z}{f}\left(\frac{5}{2}-\frac{z^{2}}{f}\right)-\frac{3}{2} \ln \frac{z+z}{a+b}\right\}\right]$,

where

$$
z=\sqrt{ }\left(z^{2}-f^{2}\right), \quad f^{i}=a^{2}-b^{2}, \quad d^{2}=a^{2}+b^{2} .
$$

Introducing $(+. i د),(.1 ?$; and $(4.18)$ in (2.4) we get, using (3.14a)

$$
\begin{aligned}
k[w]_{1}^{2} & =\operatorname{Re}\left[\left(r^{2}+\frac{d^{2}}{4}\right) \ln \frac{z+z}{a+b}+\frac{1}{3}\left\{\frac{d^{2} z}{2 f^{2}}\left(\frac{z^{2}}{f}-\frac{5}{2}\right)-\left(2 \bar{z}+\frac{r^{2} z}{f^{2}}\right)\right\} z\right. \\
& \left.-\frac{r^{4}}{8 a b}+a b\left(\frac{1}{4}+\frac{z^{2}}{f^{2}}\right)+\frac{d^{2} r^{2} z^{2}}{6 a b f^{2}}-\frac{1}{3}\left(\frac{a b}{f}+\frac{1}{8 a b}\right) z^{4}\right],
\end{aligned}
$$

where

$$
k=8 \pi \mathrm{D} / \mathrm{L} \text {. }
$$

If $\phi$ is the eccentric angle of any point $z$ on $\Gamma$ then

$$
z=a \cos \psi+i b \sin \phi, z=b \cos \phi+i a \sin \phi,
$$

and it is checked that the expression between the square brackets in (4.20) vanishes along ' ' as it siould. It is to be noted that $z=\sqrt{ }\left(z^{2}-f^{2}\right)$ is not uniform in region 1 while it is uniform in region 2. In fact, the two branches of $z$ interchange when $z$ traces a closed path $r o 1 \ldots$ any of the two foci $( \pm f, 0)$ of the ellipse. Thus, the terms containin。 $Z$ in (4.20) should appear in $w_{2}$ and not in $w_{1}$. It is also known that the singular part of the deflection $w$ at any point $P$ near a 
downward concentrated force $F$ is

$$
\mathrm{w}_{\text {sin }}=\frac{\mathrm{F}}{8 \pi \mathrm{D}} \mathrm{R}^{2} \ln \mathrm{R} \text {, }
$$

where $R$ is the distance between $P$ did the point of application of the force. Guided by these remarks and using $(-.20)$, we assume that

$$
\begin{aligned}
& k w_{1}=\frac{r^{4}}{6 a b}-a b\left(\frac{1}{4}+\frac{r^{2}}{L^{2}} \cos 2 u\right)-\frac{d^{2} r^{4}}{6 a b f^{2}} \cos 2 \theta+\frac{1}{3}\left(\frac{a b}{f}+\frac{1}{8 a b}\right) r^{4} \cos 4 \\
& -S+{ }_{0}^{\infty}\left(A_{n}+C_{n} r^{2}\right) r^{2 n} \cos 2 n y \\
& k w_{2}=\left(r^{2}+\frac{1}{4} d^{2}\right) \ln \left|\frac{z+z}{a+b}\right|+\frac{1}{3} \operatorname{Re}\left\{\frac{d^{2} z}{2 f^{2}}\left(\frac{z^{2}}{f^{2}}-\frac{5}{2}\right)-\left(2 \bar{z}+\frac{r^{2} z}{f^{2}}\right)\right\} z \\
& -S+i_{0}^{i}\left(A_{n}+C_{n} r^{2}\right) r^{2 n} \cos 2 n j,
\end{aligned}
$$

where

$$
\mathrm{S}=\frac{1}{4} \sum_{\lambda=1}^{4} \mathrm{R}_{\lambda}^{2} \ln \mathrm{R}_{\lambda}
$$

$R_{1}^{2}=r^{2}+s^{2}-2 s r \cos (\theta-\gamma), \quad R_{2}^{2}=r^{2}+s^{2}+2 s r \cos (\theta+\gamma)$,

$\mathrm{k}_{3}^{2}=\mathrm{r}^{2}+\mathrm{s}^{2}+2 \mathrm{sr} \cos (j-\gamma), \mathrm{R}_{4}^{2}=\mathrm{r}^{2}+\mathrm{s}^{2}-2 \mathrm{sr} \cos (\theta+\gamma)$,

and $A_{n}, C_{n}(n=0,1,2, \ldots)$ are real constants to be determined. It is now easily seen that the expressions $(4.23)$ and $(4.24)$ for $w_{1}$ and $w_{2}$ satisfy the biharmonic equation ( $2 . L$ ) corresponding to the load intensities $p_{1}=p_{0}$ and $p_{2}=0$, satisfy the required transition conditions along $\Gamma$ and exhibit the appropriate singular behaviour at the four points of support. The unkown constants $A_{n}(n=1,2, \ldots)$ and $C_{n}(n=0,1,2, \ldots)$ will now be deterinined from the boundary conditions (2.10). The conuition of zero deflection at any point of support serves to $f$ ind $A_{0} \cdot$ To achieve this goal, all the terms in $(4.24)$ will be explicitly expressed in terms of biharmonic functions of $r$ and $\theta$ of the types

$$
r^{-n} \cos n \dot{s i n} \quad \text { and } r^{2 \pm n} \cos n \theta \text { in }
$$

Assuming that $\psi(z, f)=\ln \left(z+\sqrt{\left(z^{2}-f^{2}\right)}\right)$ we have

$\psi^{\prime}(\mathrm{z}, \mathrm{f})=\left(\mathrm{z}^{2}-\mathrm{f}^{2}\right)^{-\frac{1}{2}}=\frac{1}{\mathrm{z}}\left(1-\frac{\mathrm{f}^{2}}{\mathrm{z}^{2}}\right)^{-\frac{1}{2}}=\frac{1}{\mathrm{z}} \sum_{0}^{\infty}\left(\mathrm{n}^{-\frac{1}{2}}\right)\left(-\frac{\mathrm{f}^{2}}{\mathrm{z}^{2}}\right)^{\mathrm{n}}\left(\left|\frac{\mathrm{z}}{\mathrm{f}}\right|>1\right)$,

where, with the usual notation

$$
\left(n^{-\frac{1}{2}}\right)=(-1)^{n} 2^{-2 n}\left(\begin{array}{c}
2 n \\
n
\end{array}\right)
$$

Integrating (4.27a), using $(4.27 b)$ and noting that $\psi(z, 0)=\ln (2 z)$ we get

$$
\psi(z, f)=\ln (2 z)-\frac{1}{2} \quad i_{1} \frac{1}{n}\left(\begin{array}{c}
2 n \\
n
\end{array}\right)\left(\frac{f}{2 z}\right)^{2 n}(|z| \geq f) .
$$

Similarly, we obtain

$$
\psi(z, f)=\ln (i f)-2 i \sum_{0}^{\infty} \frac{1}{2 n+1}\left(\begin{array}{c}
2 n \\
n
\end{array}\right)\left(\frac{z}{2 f}\right)^{2 n+1}(|z| \leq f) .
$$


Thus we have

$$
\begin{aligned}
& \ln \left|\frac{z+z}{a+b}\right|=\ln \frac{2 r}{d+b}-\frac{1}{2} \sum_{1}^{\infty} \frac{1}{n}\left(\begin{array}{c}
2 n \\
n
\end{array}\right)\left(\frac{f}{2 r}\right)^{2 n} \cos 2 n \theta(r \geq f), \\
& \ln \left|\frac{z+z}{\mid a+b}\right|=\ln \frac{f}{a+b}+2 \sum_{0}^{\infty} \frac{1}{2 n+1}\left(\begin{array}{c}
2 n \\
n
\end{array}\right)\left(\frac{r}{2 f}\right)^{2 n+1} \sin (2 n+1) \theta(r \leq f) .
\end{aligned}
$$

Expanding $\left(1-f^{2} / z^{2}\right)^{\frac{1}{2}}$ by the binomial theorem we find for $|z|<f$ :

$\frac{1}{3} \operatorname{Re}\left\{\frac{\mathrm{d}^{2} z}{2 \mathrm{f}^{2}}\left(\frac{\mathrm{z}^{2}}{\overline{\mathrm{f}}^{2}}-\frac{j}{2}\right)-\left(2 \bar{z}+\frac{\mathrm{r}^{2} \mathrm{z}}{\mathrm{f}^{2}}\right)\right\} z=\frac{3}{16} \mathrm{~d}^{2}-\frac{1}{2} \mathrm{r}^{2}-\frac{\mathrm{r}^{2}}{\mathrm{f}^{2}}\left(\frac{1}{3} \mathrm{r}^{2}+\frac{1}{2} \mathrm{~d}^{2}\right) \cos 20$

$+\frac{d^{2} r^{4}}{6 \Gamma^{4}} \cos 4 \theta+\frac{1}{2} \sum_{1}^{\infty} \frac{(f / 2 r)^{2 n}}{n+1}\left(\begin{array}{c}2 n \\ n\end{array}\right)\left\{\frac{2 n+1}{2 n-1} r^{2}+\frac{n+3}{4(n+2)} d^{2}\right\} \cos 2 n \theta \quad(r>f)$.

Similarly, writing $Z=$ if $\left(1-z^{2} / f^{2}\right)^{\frac{1}{2}}$ and expanding, we obtain the following expression for the left side of (4.29a) when $|z|<f$ :

$\frac{r}{3 f}\left(\frac{5}{4} d^{2}-2 f^{2}\right) \sin 0-\sum_{1}^{\infty} \frac{(r / 2 f)^{2 n+1}}{2 n-1}\left(\begin{array}{c}2 n \\ n\end{array}\right)\left\{\frac{2 n r^{2}}{n+1}+\frac{2 n-5}{2(2 n-3)} d^{2}\right\} \sin (2 n+1) \theta(r<f)$.

Substitution from $(4.28 \mathrm{a}, \mathrm{b})$ and $(4.29 \mathrm{a}, \mathrm{b})$ in $(4.24)$ gives

$k w_{2}=\frac{3 d^{2}}{16}-\frac{1}{2} r^{2}+\left(r^{2}+\frac{1}{4} d^{2}\right) \ln \frac{2 r}{a+b}-\frac{r^{2}}{f^{2}}\left(\frac{1}{3} r^{2}+\frac{1}{2} d^{2}\right) \cos 2 \theta+\frac{d^{2} r^{4}}{6 f^{4}} \cos 4 u$

$-S+\sum_{1}^{\infty} \frac{(f / 2 r)^{2 n} \cos 2 n u}{4 n(n+1)}\left(\begin{array}{c}2 n \\ n\end{array}\right)\left(\frac{2 r^{2}}{2 n-1}-\frac{d^{2}}{n+2}\right)+\sum_{0}^{\infty}\left(A_{n}+C_{n} r^{2}\right) r^{2 n} \cos 2 n \theta \quad(r \geq f)$,

$k w_{2}=\frac{r}{f}\left(r^{2}+\frac{4}{3} b^{-}\right) \sin \theta+\left(r^{2}+\frac{1}{4} d^{2}\right) \ln \frac{f}{a+b}-s$

$+2 \sum_{1}^{\infty} \frac{(r / 2 f)^{2 n+1}}{4 n^{2}-1}\left(\begin{array}{c}2 n \\ n\end{array}\right)\left(\frac{2 d^{2}}{2 n-3}-\frac{r^{2}}{n+1}\right) \sin (2 n+1) \theta+\sum_{0}^{\infty}\left(A_{n}+C_{n} r^{2}\right) r^{2 n} \cos 2 n \theta(r \leq f)$.

It can he easily shown that for $r \geq s$

$$
\begin{aligned}
R_{\lambda}^{2} \ln R_{\lambda} & =\left(r^{2}+s^{2}\right) 1 n r+s^{2}-s r\left(1+21 n r+\frac{s^{2}}{2 r^{2}}\right) \cos \left(\theta-\gamma_{\lambda}\right) \\
& +\sum_{2}^{\infty} \frac{1}{n}\left(\frac{r^{2}}{n-1}-\frac{s^{2}}{n+1}\right)\left(\frac{s}{r}\right)^{n} \cos n\left(\theta-\gamma_{\lambda}\right)
\end{aligned}
$$

and

$s=\left(r^{2}+s^{2}\right) \ln r+s^{2}+\sum_{2}^{\infty} \frac{1}{\angle n}\left(\frac{r^{2}}{2 n-1}-\frac{s^{2}}{2 n+1}\right)\left(\frac{s}{r}\right)^{2 n} \cos 2 n \gamma \cos 2 n \theta(r \geq s)$.

For $r=s$ we interchange $r$ and $s$ in $(4.31 \mathrm{a}, \mathrm{b})$.

Introducing $(4.31 \mathrm{~b})$ in $(4.30 \mathrm{a})$ yields

$$
k w_{2}=\sum_{0}^{\infty} L_{n}(r) \cos 2 n \theta,
$$

where $r \geq$ the greater of $f$ and $s$,

$L_{0}(r)=A_{0}^{\prime}+B_{0}^{\prime} \ln r+C_{0}^{\prime} r^{2}, L_{n}(r)=A_{n}^{\prime} r^{2 n}+B_{n}^{\prime} r^{-2 n}+C_{n}^{\prime} r^{2+2 n}+D_{n}^{\prime} r^{2-2 n}(n \geq 1)$,

$A_{j}^{\prime}=A_{0}-s^{2}+\frac{d^{2}}{4}\left(\frac{3}{4}+\ln \frac{2}{a+b}\right), B_{j}^{\prime}=\frac{1}{4} d^{2}-s^{2}, C_{0}^{\prime}=C_{0}-\frac{1}{2}+\ln \frac{2}{a+b}$, 
$A_{1}^{\prime}=A_{1}-\frac{d^{2}}{2 f^{2}}, C_{1}^{\prime}=C_{1}-\frac{1}{3 f^{2}}, A_{2}^{\prime}=A_{2}+\frac{d^{2}}{6 f 4}$,

$A_{n}^{\prime}=A_{n}(n \geq 3), B_{n}^{\prime}=\frac{s^{2 n+2} \cos 2 n \gamma}{2 n(2 n+1)}-\frac{d^{2}(f / 2)^{2 n}}{4 n(n+1)(n+2)}\left(\begin{array}{c}2 n \\ n\end{array}\right)(n \geq 1)$,

$C_{n}^{\prime}=C_{n}(n \geq 2), D_{n}^{\prime}=-\frac{s^{2 n} \cos 2 n \gamma}{2 n(2 n-1)}+\frac{(f / 2)^{2 n}}{2 n(n+1)(2 n-1)}\left(\begin{array}{c}2 n \\ n\end{array}\right)(n \geq 1)$.

Applying tue differential operators (2.11) and (2.12) to the functions in (4.32) gives

$f_{r}\left(d, d^{\prime}\right)\left\{l_{U}(r)\right\}=O\left[(k-1) C_{j}^{\prime}-r^{-2} B_{u}^{\prime}\right], I_{r}\left(d, d^{\prime}\right)\left\{L_{0}(r)\right\}=0$,

$f_{r}\left(d, d^{\prime}\right)\left\{L_{n}(r) \cos \left\langle n y !=2 n \sigma\left[(2 n-1) \cdot 1_{n}^{\prime} r^{2 n-2}+(2 n+1) B_{n}^{\prime} r^{-2 n-2}\right.\right.\right.$

$\left.+\left(1+\frac{1}{2 n}\right)(2 n+k-1) C_{n}^{\prime} r^{2 n}+\left(1-\frac{1}{2 n}\right)(2 n-k+1) D_{n}^{\prime} 2^{-2 n}\right] \cos 2 n \theta$,

$\mathrm{rF}_{\mathrm{r}}\left(\mathrm{d}, \mathrm{d}^{\prime}\right)\left\{\mathrm{L}_{\mathrm{n}}(\mathrm{r}) \cos 2 \mathrm{n} \theta\right\}=-4 \mathrm{n}^{2} \sigma\left[(2 n-1) A_{n}^{\prime} \mathrm{r}^{2 n-2}-(2 n+1) B_{n}^{\prime} r^{-2 n-2}\right.$

$\left.+\left(1+\frac{1}{2 n}\right)(2 n-k-1) C_{n}^{\prime} r^{2 n}-\left(1-\frac{1}{2 n}\right)(2 n+k+1) D_{n}^{\prime} r^{-2 n}\right] \cos 2 n \theta$,

where

$$
\sigma=1-v, \quad k=(3+v) /(1-v) .
$$

Inserting (4.32) in the boundary conditions (2.10), using (4.33)-(4.40), equating the coefficients of $\cos 2 n \theta(n=0,1,2, \ldots)$ in the resulting identities to zero and solvin: the obtained systems of linear equations we find

$C_{u}^{\prime}=\frac{1}{8} B\left(v^{2}-4 u^{2}\right), C_{0}=\frac{1}{2}+\frac{1}{8} B\left(v^{2}-4 u^{2}\right)+\ln \frac{a+b}{2}$,

$A_{n}^{\prime}=\frac{c^{2-2 n}}{2 n \kappa}\left[\left\{u^{2}-\frac{4 n^{2}+k^{2}-1}{2 n(2 n-1)}\right\} u^{2 n} \cos 2 n \gamma+\frac{(f / 2 c)^{2 n}}{2 n+2}\left(\begin{array}{c}2 n \\ n\end{array}\right)\left\{\frac{4 n^{2}+\kappa^{2}-1}{n(2 n-1)}-\frac{2 n+1}{n+2} v^{2}\right\}\right](n \geq 1)$,

$C_{n}^{\prime}=\frac{c^{-2 n}}{2 n k}\left[\left(1-\frac{2 n u^{2}}{2 n+1}\right) u^{2 n} \cos 2 n \gamma+\frac{(f / 2 c)^{2 n}}{n+1}\left(\begin{array}{c}2 n \\ n\end{array}\right)\left(\frac{n v^{2}}{n+2}-1\right)\right] \quad(n \geq 1)$,

where

$$
B=(1-v) /(1+v), \quad u=s / c, \quad v=d / c .
$$

When the values of $A_{1}, A_{2}$ and $C_{1}$ are replaced by their values in terms of $A_{1}^{\prime}, A_{2}^{\prime}$ and $C_{1}^{\prime}$ by means of (4.35) it is found that (4.23) and (4.30a) take the forms

$$
\begin{aligned}
k w_{1} & =\frac{a-b}{2(a+b)}\left(1-\frac{r^{2}}{3 a b}\right) r^{2} \cos 2 \theta+\frac{r^{4}}{8 a b}\left\{1+\frac{1}{3}\left(\frac{a-b}{a+b}\right)^{2} \cos 4 \theta\right\}-\frac{1}{4} a b-s \\
& +A_{0}+C_{U} r^{2}+\sum_{1}^{\infty}\left(A_{n}^{\prime}+C_{n}^{\prime} r^{2}\right) r^{2 n} \cos 2 n \theta, \\
k w_{2} & =\frac{3}{16} d^{2}-\frac{1}{2} r^{2}+\left(r^{2}+\frac{1}{4} d^{2}\right) 1 n \frac{2 r}{a+b}+\frac{1}{4} \sum_{1}^{\infty}\left(\begin{array}{c}
2 n \\
n
\end{array}\right) \frac{(f / 2 r)}{n(n+1)}\left(\frac{2 n}{2 n-1}-\frac{d^{2}}{n+2}\right) \cos 2 n \theta-s \\
& +A_{U}+C_{U} r^{2}+\underset{1}{2}\left(A_{n}^{\prime}+C_{n}^{\prime} r^{2}\right) r^{2 n} \cos 2 n \theta(r=f),
\end{aligned}
$$

where $C_{U}$ is given by $(4.4 \angle a)$ and $A_{n}^{\prime}, C_{n}^{\prime}$ are given by $(4.42 b, c)$. For points of region 2 at which $\mathrm{r} \leq \mathrm{f}$ the deflection $\mathrm{w}_{2}$ is furnished by (4.30b). It is easily 
seen that such points exist only if $f \geq b$, i.e., if the eccentricity of the ellipse $\geq \sqrt{2} / 2$. In any case, $w_{2}$ is given by (4.24). The constant $A_{0}$ can always be determined from the condition that the deilection vanishes at any of the four points of support. If all these points lie in the loaded region then (4.44) gives $A_{0}=\frac{1}{4} a b+s^{2}\left[\sin ^{2} \gamma \ln \sin \gamma+\cos ^{2} \gamma \ln \cos \gamma-\frac{1}{2}+\ln \frac{8 s^{2}}{a+b}+\frac{1}{8} \beta\left(4 u^{2}-v^{2}\right)\right.$ $\left.+\frac{a-b}{2(d+b)}\left(\frac{s^{2}}{3 a b}-1\right) \cos 2 \gamma-\frac{s^{2}}{8 a b}\left\{1+\frac{1}{3}\left(\frac{a-b}{a+b}\right)^{2} \cos 4 \gamma\right\}\right]-\sum_{1}^{\infty}\left(A_{n}^{\prime}+C_{n}^{\prime} s^{2}\right) s^{2 n} \cos 2 n \gamma$. For a single support at the centre we have $s=0, u=0, A_{0}=\frac{1}{4}$ ab and $s=r^{2} \ln r$. If all the survort soints 1 ie in the unloaded region, then either (4.45) or (4.30b) can be used to determine $A_{0}$ according as $s \geq f$ or $s \leq f$. If $s \geq f$ we have

$$
\begin{aligned}
A_{0} & =s^{2}\left[\sin ^{2} \gamma \ln (' s \sin \gamma)+\cos ^{2} \gamma \ln (4 s \cos \gamma)+\frac{1}{\delta} \beta\left(4 u^{2}-v^{2}\right)\right] \\
& -\frac{d^{2}}{4}\left(\frac{3}{4}+\ln \frac{2 s}{a+b}\right)+i_{1}^{\infty}\left\{\frac{(f / 2 s)^{2 n}}{4 n(n+1)}\left(\begin{array}{c}
2 n \\
n
\end{array}\right)\left(\frac{d^{2}}{n+2}-\frac{2 s^{2}}{2 n-1}\right)-\left(A_{n}^{\prime}+C_{n}^{\prime} s^{2}\right) s^{2 n}\right\} \cos 2 n \gamma,
\end{aligned}
$$

but if $s \leq f$ then (4.30b) gives

$$
\begin{aligned}
A_{O} & =s^{2}\left[\sin ^{2} \gamma \ln \sin \gamma+\cos ^{2} \gamma \ln \cos \gamma-\frac{1}{2}+\ln \frac{8 s^{2}}{f}+\frac{1}{8} B\left(4 u^{2}-v^{2}\right)\right] \\
& -\frac{d^{2}}{4} \ln \frac{f}{a+b}-\frac{s}{f}\left(s^{2}+\frac{4}{3} b^{2}\right) \sin \gamma+2 \sum_{1}^{\infty} \frac{(s / 2 f)}{4 n^{2}-1}\left(\begin{array}{c}
2 n \\
n
\end{array}\right)\left(\frac{s^{2}}{n+1}-\frac{2 d^{2}}{2 n-3}\right) \sin (2 n+1) \gamma \\
& -\sum_{1}^{\infty}\left(A_{n}+C_{n} s^{2}\right) s^{2 n} \cos 2 n \gamma .
\end{aligned}
$$

In any case, the deflection at the centre of the plate is

$$
w_{0}=\frac{L}{\delta \pi D}\left(A_{0}-\frac{1}{4} a b-s^{2} \ln s\right),
$$

where the appropriate value of $A_{0}$ is taken.

\section{BOUNDARY AND CENTRAL VALUES OF MOMENTS AND SHEARS.}

It can be easily shown that the deflections (4.23) and (4.24) may be written in the forms

$$
\begin{aligned}
& w_{1}=2 \operatorname{Re}\left[\bar{z} \Omega_{1}(z)+w_{1}(z)\right]+w_{1}(z, \bar{z}), \\
& w_{2}=2 \operatorname{Re}\left[\bar{z} s_{2}(z)+w_{2}(z)\right],
\end{aligned}
$$

wherc

$$
\begin{aligned}
& 2 k s_{1}(z)=-\frac{d^{2} z^{3}}{6 a b f^{2}}-\frac{1}{4} \sum_{1}^{4} z_{\lambda} \ln z_{\lambda}+\sum_{0}^{\infty} C_{n} z^{2 n+1}, \\
& 2 k \omega_{1}(z)=\frac{1}{3}\left(\frac{a b}{f^{4}}+\frac{1}{8 a b}\right) z^{4}-a b\left(\frac{1}{4}+\frac{z^{2}}{f^{2}}\right)+\frac{1}{4} \sum_{1}^{4} \bar{z}_{\lambda} z_{\lambda} \ln z_{\lambda}+\sum_{0}^{\infty} A_{n} z^{2 n}, \\
& 2 k s_{2}(z)=z \ln \frac{z+z}{a+b}-\frac{1}{3}\left(2+\frac{z^{2}}{f^{2}}\right) z-\frac{1}{4} \sum_{1}^{4} z_{\lambda} \ln z_{\lambda}+\sum_{0}^{\infty} C_{n} z^{2 n+1}, \\
& 2 k \omega_{2}(z)=\frac{d^{2}}{4}\left\{\frac{2 z L}{3 f^{2}}\left(\frac{z^{2}}{f^{2}}-\frac{5}{2}\right)+\ln \frac{z+z}{a+b}\right\}+\frac{1}{4} \sum_{1}^{4} \bar{z}_{\lambda} z_{\lambda} \ln z_{\lambda}+\sum_{0}^{\infty} A_{n} z^{2 n},
\end{aligned}
$$


$z_{\lambda}=z-z_{\lambda}$ and the real constants $A_{n}, C_{n}$ have been determined in the previous section. The moments and shears at any point of the plate can be obtained either by substitution from $(4.15),(j .51 a, b)$ and $(5.52 a, b)$ in $(2.7 a, b)$ and (2.8) or by introducing $(4.44),(4.45),(4.30 b)$ in $(2.5 a, b, c)$ and $(2.6)$, noting that $S$ is defined by (4.25), (4.26) and its expansion is (4.31b) if $r \geq s$ and interchanging $r, s$ in (4.31b) if $r \leq s$. After extensive algebraic manipulation, it is found that $\left(M_{r}\right)_{r=c}=0$ as expected and

$$
\begin{aligned}
& \left(M_{U}\right)_{r=c}=-\frac{(1+v) L}{4 \pi k}\left[\beta k\left(\frac{1}{4} v^{2}-u^{2}\right)+\sum_{1}^{\infty}\left(2-2 u^{2}+\frac{k+1}{n}\right) u^{2 n} \cos 2 n \gamma \cos 2 n \theta\right. \\
& \left.+i_{1}^{\infty}\left(\begin{array}{c}
2 n \\
n
\end{array}\right) \frac{(f / 2 c)^{2 n}}{n+1}\left(\frac{2 n+1}{n+2} v^{2}-\frac{2 n+k+1}{n}\right) \cos 2 n \theta\right] \text {, } \\
& \left(M_{r \cdot}\right)_{r=c}=\frac{L}{4 \pi c} \sum_{1}^{\infty}\left(2 u^{2}-2+\frac{k-1}{n}\right) u^{2 n} \cos 2 n \gamma \sin 2 n \theta \\
& \left.+i_{1}^{\infty}\left(\begin{array}{c}
2 n \\
n
\end{array}\right) \frac{(f / 2 c)^{2 n}}{n+1}\left(2-\frac{k-1}{n}-\frac{2 n+1}{n+2} v^{2}\right) \sin 2 n \theta\right] \text {, } \\
& \left(u_{r}\right)_{r=c}=\frac{L}{2 \pi k c} \int_{1}^{\infty}\left(2 n u^{2}-2 n+k-1\right) u^{2 n} \cos 2 n \gamma \cos 2 n \theta \\
& \left.+\sum_{1}^{\infty}\left(\begin{array}{c}
2 n \\
n
\end{array}\right) \frac{(\dot{r} / 2 c)^{2 n}}{n+1}\left\{2 n-k+1-\frac{n(2 n+1)}{n+2} v^{2}\right\} \cos 2 n \theta\right] \text {, } \\
& \left(U_{\theta}\right)_{r=c}=\frac{L}{2 \pi k c}\left[\sum_{1}^{\infty}\left(2 n+k+1-2 n u^{2}\right) u^{2 n} \cos 2 n \gamma \sin 2 n \theta\right. \\
& \left.-\sum_{1}^{\infty}\left(\begin{array}{c}
2 n \\
n
\end{array}\right) \frac{(f / 2 c)^{2 n}}{n+1}\left\{2 n+k+1-\frac{n(2 n+1)}{n+2} v^{2}\right\} \text { sin } 2 n \theta\right\} \text {. }
\end{aligned}
$$

It is easily seen that (5.53b), (5.54a) satisfy the second boundary condition in (2.9) and $(5.33 a)$, (j.54b) satisfy (2.13).

All the infinite series appearing in this section and in section 4 are convergent in the intervals mentioned and some of them will be summed in section 8 .

The following formulae are obtained for the moments and shears at the centre:

$$
\begin{gathered}
\left.M_{r}\right)_{U}=\frac{L}{8 \pi}\left[(1+v)\left\{\beta\left(u^{2}-\frac{1}{4} v^{2}\right)+1+2 \ln \frac{2 s}{a+b}\right\} \pm(1-v)\{\cos 2 \gamma\right. \\
\left.\left.\left.-\frac{a-b}{a+b}+\frac{u^{2}}{2 k}\left(K_{\theta}\right)_{0}^{2}+3-2 u^{2}\right) \cos 2 \gamma+\frac{f^{2}}{8 k c^{2}}\left(v^{2}-\kappa^{2}-3\right)\right\} \cos 2 \theta\right], \\
\left(M_{r \theta}\right)_{U}=\frac{(1-v) L}{8 I i}\left[\cos 2 \gamma-\frac{a-b}{a+b}+\frac{u^{2}}{2 k}\left(k^{2}+3-2 u^{2}\right) \cos 2 \gamma+\frac{f^{2}}{8 k c^{2}}\left(v^{2}-\kappa^{2}-3\right)\right] \sin 2 \theta . \\
\left(Q_{r}\right)_{0}=\left(Q_{\theta}\right)_{0}=0 .
\end{gathered}
$$

6. INF INITE PLATE UNIFORMLY LOADEY OVER AN ELLIPTIC PATCH AND SUPPORTED ON COLUMNS. Letting $c \rightarrow \infty$ in $(4.44),(4.45)$ and $(4.30 b)$ leads to

$$
\begin{aligned}
k w_{1} & =A_{u}-\frac{1}{4} a b-s+\left(\frac{1}{2}+\ln \frac{a+b}{2}\right) r^{2}+\frac{a-b}{2(a+b)}\left(1-\frac{r^{2}}{3 a b}\right) r^{2} \cos 2 \theta \\
& +\frac{r^{4}}{3 a b}\left[1+\frac{1}{3}\left(\frac{a-b}{a+b}\right)^{2} \cos 40\right]
\end{aligned}
$$




$$
\begin{aligned}
k w_{2} & =A_{U}+\frac{d^{2}}{4}\left(\frac{3}{4}+\ln \frac{2 r}{a+b}\right)+r^{2} \ln r-s \\
& +\sum_{1}^{\infty} \frac{(f / 2 r)}{4 n(n+1)}\left(\begin{array}{c}
2 n \\
n
\end{array}\right)\left(\frac{2 r^{2}}{2 n-1}-\frac{d^{2}}{n+2}\right) \cos 2 n \theta(r \geq f), \\
k w_{2} & =A_{0}+\left(\frac{1}{2}+\ln \frac{f}{2}\right) r^{2}+\frac{1}{4} d^{2} \ln \frac{f}{a+b}+\frac{r}{f}\left(r^{2}+\frac{4}{3} b^{2}\right) \sin \theta+\frac{r^{2}}{f^{2}}\left(\frac{1}{3} r^{2}+\frac{1}{2} d^{2}\right) \cos 2 \theta \\
& -\frac{d^{2} r^{4}}{6 f^{4}} \cos 4 u-s+2 \sum_{1}^{\infty} \frac{(r / 2 f)^{2 n+1}}{4 n^{2}-1}\left(\begin{array}{c}
2 n \\
n
\end{array}\right)\left(\frac{2 d^{2}}{2 n-3}-\frac{r^{2}}{n+1}\right) \sin (2 n+1) \theta(r \leq f),
\end{aligned}
$$

where

$$
\begin{aligned}
A_{0}=\frac{1}{4} a b & +s^{2}\left[\sin ^{2} \gamma \ln \sin \gamma+\cos ^{2} \gamma \ln \cos \gamma-\frac{1}{2}+\ln \frac{8 s^{2}}{a+b}\right. \\
& \left.+\frac{a-b}{2(a+b)}\left(\frac{s^{2}}{3 a b}-1\right) \cos 2 \gamma-\frac{s^{2}}{3 a b}\left\{1+\frac{1}{3}\left(\frac{a-b}{a+b}\right)^{2} \cos 4 \gamma\right\}\right],
\end{aligned}
$$

if the supports lie in the loaded region;

$$
\begin{aligned}
A_{0} & =s^{2} \sin ^{2} \gamma \ln (4 s \sin \gamma)+s^{2} \cos ^{2} \gamma \ln (4 s \cos \gamma)-\frac{d^{2}}{4}\left(\frac{3}{4}+\ln \frac{2 s}{a+b}\right) \\
& +\sum_{1}^{\infty} \frac{(f / 2 s)^{2 n}}{4 n(n+1)}\left(\begin{array}{c}
2 n \\
n
\end{array}\right)\left(\frac{d^{2}}{n+2}-\frac{2 s^{2}}{2 n-1}\right) \cos 2 n \gamma,
\end{aligned}
$$

if the supports 1 ie in the unloaded region and $\mathrm{s} \geq \mathrm{f}$;

$$
\begin{aligned}
A_{0} & =s^{2}\left\{\sin ^{2} \gamma \ln \sin \gamma+\cos ^{2} \gamma \ln \cos \gamma+\ln \frac{3 s^{2}}{\dot{f}}-\frac{1}{2}\right\}+\frac{d^{2}}{4} \ln \frac{a+b}{f} \\
& -\frac{s}{\mathrm{f}}\left(s^{2}+\frac{4}{3} b^{2}\right) \sin \gamma-\frac{s^{2}}{f^{2}}\left(\frac{s^{2}}{3}+\frac{d^{2}}{2}\right) \cos 2 \gamma+\frac{d^{2} s^{4}}{6 f^{4}} \cos 4 \gamma \\
& +2 \sum_{1}^{\infty} \frac{(f / 2 s)^{2 n+1}}{4 n^{2}-1}\left(\begin{array}{c}
2 n \\
n
\end{array}\right)\left(\frac{s^{2}}{n+1}-\frac{2 d^{2}}{2 n-3}\right) \sin (2 n+1) \gamma,
\end{aligned}
$$

if the supports lie in the unloaded region and $s \leq f$.

At the centre of the ellipse the moments $(5.55 \mathrm{a}, \mathrm{b})$ reduce to

$$
\begin{aligned}
& \left(M_{r}\right)_{0}=\frac{L}{i \pi}\left[(1+v)\left\{1+2 \ln \frac{2 s}{a+b}\right\} \pm(1-v)\left\{\cos 2 \gamma-\frac{a-b}{a+b}\right\} \cos 2 \theta\right], \\
& \left.(M)_{0}\right] \\
& \left(M_{r \theta}\right)_{0}=\frac{(1-v) L}{3 \pi}\left[\cos 2 \gamma-\frac{a-b}{a+b}\right] \sin 20 .
\end{aligned}
$$

\section{THIN CIRCULAR PLATL UNDER A VARIABLE LINE LOADING ALONG A DIAMETER AND SUPPORTED ON COLUMNS.}

When the minor axis of the loaded elliptic patch $\rightarrow 0$ we have the case of a variable line loading extending along tue $x$-axis from $x=-a$ to $x=a$. If $b \rightarrow 0$ and $\mathrm{p}_{0} \rightarrow \infty$ such that $2 \mathrm{bp}_{0} \rightarrow \mathrm{p}_{1}$ then the intensity of this 1 ine loading at a distance $x$ from the centre equals $p_{1} \sqrt{ }\left(1-x^{2} / a^{2}\right)$. Deflections, moments and shears corresponding to this case can be dedcued from those for region 2 in sections 4,5 by setting $b=0, d=f=a, L=\frac{1}{2} \pi a p_{1}$ and noting that separate expressions are

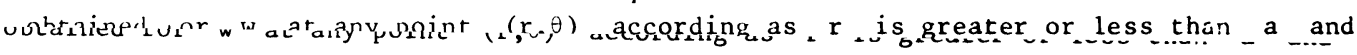
tnc tolumns lie outside or inside the circle $r=a$. 
8. THIN CIRCULAR PLATE UNIFORMLY LOADED OVER A CONCENTRIC CIRCULAR PATCH AND SUPPORTED O.N COLUMNS.

Setting $b=a, f=0$ in $(4.44),(4.45)$ and $(4.42 a, b, c)$ we get

$$
\begin{aligned}
k w_{1}=A_{0} & -\frac{1}{4} a^{2}+\left\{\frac{1}{2}+\ln a+\frac{1}{4} B\left(t^{2}-2 u^{2}\right)\right\} r^{2}+\frac{r^{4}}{8 a^{2}}-s \\
& +\sum_{n}^{\infty}\left(A_{n}^{\prime}+C_{n}^{\prime} r^{2}\right) r^{2 n} \cos 2 n \theta, \\
k w_{2}=\Lambda_{u} & +\frac{3}{8} a^{2}+\left\{1 n a+\frac{1}{4} B\left(t^{2}-2 u^{2}\right)\right\} r^{2}+\left(r^{2}+\frac{1}{2} a^{2}\right) \ln \frac{r}{a}-s \\
& +\sum_{1}^{\infty}\left(A_{n}^{\prime}+C_{n}^{\prime} r^{2}\right) r^{2 n} \cos 2 n \theta,
\end{aligned}
$$

where $t=a / c$ and

$$
\begin{aligned}
A_{n}^{\prime}= & \frac{c^{2-2 n} u^{2 n}}{2 n k}\left\{u^{2}-\frac{4 n^{2}+k^{2}-1}{2 n(2 n-1)}\right\} \cos 2 n \gamma, C_{n}^{\prime}=\frac{c^{-2 n} u^{2 n}}{2 n k}\left(1-\frac{2 n u^{2}}{2 n+1}\right) \cos 2 n \gamma, \\
A_{0}= & 4^{2} a^{2}+s^{2}\left[\sin ^{2} \gamma 1 n \sin \gamma+\cos ^{2} \gamma \ln \cos \gamma-\frac{1}{2}+1 n \frac{4 s^{2}}{a}+\frac{1}{4} B\left(2 u^{2}-t^{2}\right)-\frac{s^{2}}{8 a^{2}}\right] \\
& -\frac{2}{i}\left(A_{n}^{\prime}+C_{n}^{\prime} s^{2}\right) s^{2 n} \cos 2 n \gamma(s \leq a),
\end{aligned}
$$$$
A_{0}=s^{2}\left\lfloor\sin ^{2} \gamma \ln \sin \gamma+\cos ^{2} \gamma \ln \cos \gamma+\ln (4 s)+\frac{1}{4} B\left(2 u^{2}-t^{2}\right)\right\rfloor-\frac{a^{2}}{2}\left(\frac{3}{4}+\ln \frac{s}{a}\right)
$$$$
-i\left(A_{n}^{\prime}+C_{n}^{\prime} s^{2}\right) s^{2 n} \cos 2 n \gamma(s \geq a) .
$$

The infunite series in (3.62) and (8.63) equals

$$
\begin{aligned}
& \frac{r^{2}+s^{2}+\left(k^{2}-1\right) c^{2}}{2 k}\left\{J_{0}\left(\xi, \phi_{1}\right)+J_{0}\left(\xi, \phi_{2}\right)\right\}-\frac{r^{2} u^{2}}{2 k}\left\{J_{1}\left(\xi, \phi_{1}\right)+J_{1}\left(\xi, \phi_{2}\right)\right\} \\
& -\frac{1}{2} k c^{2}\left\{J_{-1}\left(\xi, \phi_{1}\right)+J_{-1}\left(\xi, \phi_{2}\right)\right\}+\frac{\left(k^{2}-1\right) c^{2}}{8 k}\left\{J\left(\xi, \phi_{1}\right)+J\left(\xi, \phi_{2}\right)\right\},
\end{aligned}
$$

where

$$
\begin{aligned}
& i_{0}=r s / c^{2}, \psi_{1}=\theta-r, \phi_{2}=\theta+\gamma, \\
& J_{C}(\xi, \dot{ })=\sum_{1}^{\infty} \frac{5^{2 n} \cos 2 n \phi}{2 n}=-\frac{1}{4} \ln \left(1+\xi^{4}-2 \xi^{2} \cos 2 \phi\right) \text {, } \\
& J_{1}\left(i_{+}\right)=\sum_{1} \frac{\xi^{2 n} \cos 2 n_{i}}{2 n+1}=\frac{1}{4 \xi}\left[\cos \phi \ln \frac{1+\xi^{2}+2 \xi \cos \phi}{1+\xi^{2}-2 \xi \cos \phi}+2 \sin \phi \tan ^{-1} \frac{2 \xi \sin \phi}{1-\xi^{2}}\right]-1 \text {, } \\
& J_{-1}(\xi, \psi)=\sum_{1}^{\infty} \frac{\xi^{2 n} \cos 2 n \phi}{2 n-1}=\frac{1}{4} \xi\left[\cos \varphi \ln \frac{1+\xi^{2}+2 \xi \cos \phi}{1+\xi^{2}-2 \xi \cos \phi}-2 \sin \phi \tan ^{-1} \frac{2 \xi \sin \phi}{1-\xi^{2}}\right] \text {, } \\
& J(\zeta, \dot{\zeta})=\sum_{1}^{\infty} \frac{\zeta^{2 \mathrm{n}} \cos 2 \mathrm{n} \psi}{\mathrm{n}^{2}}=\operatorname{Re} \sum_{1}^{\infty} \frac{\zeta^{2 \mathrm{n}}}{\mathrm{n}^{2}}=\operatorname{Re}\left\{-\int_{0}^{\zeta} \frac{\ln (1-\lambda)}{\lambda} \mathrm{d} \lambda\right\} \quad\left(\zeta=\xi \mathrm{e}^{\mathrm{i} \phi}\right) .
\end{aligned}
$$

The last function is the dilogaritnm studied in the last three references of [7]. The deflection at the centre is given by

$$
\begin{aligned}
k w_{0}=s^{2}\left\lfloor\sin ^{2} \gamma \ln \sin \gamma\right. & +\cos ^{2} \gamma \ln \cos \gamma+\ln \frac{4 s}{a}-\frac{1}{2}-\frac{s^{2}}{8 a^{2}} \\
& \left.+\frac{1}{4} b\left(2 u^{2}-t^{2}\right)\right\rfloor-\frac{c^{2} \dot{v}}{4 k}(s=a),
\end{aligned}
$$




$$
\begin{aligned}
& k w_{0}=s^{2} \quad \sin ^{2} \gamma \ln \sin \gamma+\cos ^{2} \gamma \ln \cos \gamma+\ln 4+\frac{1}{4} \beta\left(2 u^{2}-t^{2}\right) \\
& \text { where } \\
& -\frac{a^{2}}{2}\left(\frac{5}{4}+\ln \frac{s}{a}\right)-\frac{c^{2} \delta}{4 k}(s \geq a) \\
& \left.0=\left(\kappa^{2}+2 u^{2}-1\right)+2 u_{u}\left(u^{2}, 2 \gamma\right)-\ln \left(1-u^{4}\right)\right\}-2 u^{4}\left\{J_{1}\left(u^{2}, 2 \gamma\right)+\frac{1}{2 u^{2}} \ln \frac{1+u^{2}}{1-u^{2}}-1\right\} \\
& \left.-2 k^{2}\left\{i-1\left(u^{2}, 2 \gamma\right)+\frac{1}{2} u^{2} 1 u \frac{1+u^{2}}{1-u^{2}}\right\}+\frac{1}{2}\left(k^{2}-1\right)\left\{J^{2} u^{2}, 0\right)+J\left(u^{2}, 2 \gamma\right)\right\} .
\end{aligned}
$$

Setting $r=\pi / 4$ we obtain

$$
\begin{aligned}
& k w_{0}=s^{2}\left[\frac{1}{2}(3 \ln L-1)+\ln \frac{s}{a}-\frac{s^{2}}{\delta a^{2}}+\frac{1}{4} \beta\left(2 u^{2}-t^{2}\right)\right]+\frac{c^{2} \delta}{k} \quad(s \leq a), \\
& k w_{u}=s^{2}\left[\frac{3}{2} \ln L+\frac{1}{4} \beta\left(2 u^{2}-t^{2}\right)\right]-\frac{a^{2}}{2}\left(\frac{5}{4}+\ln \frac{s}{a}\right)+\frac{c^{2} \delta^{\prime}}{k} \quad(s \geq a),
\end{aligned}
$$

where

$$
\begin{aligned}
\delta^{\prime} & \left.=i_{1}^{\infty} \mid \frac{16 n^{2}+k^{2}-1}{16 n^{2}(4 n-1)}-\frac{u^{2}}{2 n}+\frac{u^{4}}{4 n+1}\right\rfloor \\
& =\frac{1-k^{2}}{10} J\left(u^{4}, 0\right)-u^{4}+\frac{1}{4}\left\{k^{2}-1+\left(k^{2}+3\right) u^{2}\right\} \ln \left(1+u^{2}\right) \\
& +\frac{1}{4}\left(\kappa^{2}+2 u^{2}-1\right) \ln \left(1+u^{4}\right)+\frac{1}{2}\left(1-k^{2}\right)\left\{u^{2} \tan ^{-1} u^{2}-\frac{1}{2}\left(1-u^{2}\right) \ln \left(1-u^{2}\right)\right\} .
\end{aligned}
$$

It is verified that $(8.71 \mathrm{a}, \mathrm{b})$ agree with (3.14) and (3.6a) of [3], noting the difference in notation. There are misprints in equation (3.5a), p.738 of [3] ard $\cos \alpha$ which appears twice in this equation must be replaced by $\cos s \alpha$.

Putting $b=a, f=0$ in $(5.53 a, b),(5.54 a, b)$ and summing the infinite series obtained we get the closed formulae

$$
\begin{aligned}
& \left(M_{\theta}\right)_{r=c}=-\frac{(1+v) L}{4 \pi k} \mid u^{2}-1+\beta k\left(\frac{1}{2} t^{2}-u^{2}\right)-\frac{1}{4}(k+1) \ln \left(I_{1} I_{2}\right) \\
& \left.+\frac{1}{2}\left(1-u^{2}\right)\left(1-u^{4}\right)\left(I_{1}^{-1}+I_{2}^{-1}\right)\right] . \\
& \left(M_{r \forall}\right)_{r=c}=\frac{L}{4 \pi k}\left[\frac{1}{i s}\left(\tan ^{-1} \frac{u^{2} \sin 2 \psi_{1}}{1-u^{2} \cos 2 \psi_{1}}+\tan ^{-1} \frac{u^{2} \sin 2 \phi_{2}}{1-u^{2} \cos 2 \phi_{2}}\right)\right. \\
& \left.-u^{2}\left(1-u^{2}\right)\left(\frac{\sin 2 \varphi_{1}}{I_{1}}+\frac{\sin 2 \psi_{2}}{I_{2}}\right)\right] \text {, } \\
& \left(Q_{r}\right)_{r=c}=\frac{L}{4 \pi K c}\left[1-K+\left(1-u^{2}\right)\left(1+u^{4}+\frac{1+u^{2}}{B}\right)\left(I_{1}^{-1}+I_{2}^{-1}\right)\right. \\
& \left.-\left(1-\mathrm{u}^{2}\right)\left(1-\mathrm{u}^{4}\right)^{2}\left(\mathrm{I}_{1}^{-2}+\mathrm{I}_{2}^{-2}\right)\right\rfloor, \\
& \left(Q_{j}\right)_{r=c}=\frac{L}{4 \pi K c} \mid(1+K) u^{2}\left(\frac{\sin 2 \phi_{1}}{I_{1}}+\frac{\sin 2 \phi_{2}}{I_{2}}\right) \\
& \left.+2 u^{2}\left(1-u^{2}\right)\left(1-u^{4}\right)\left(\frac{\sin 2 \phi 1}{I_{1}^{2}}+\frac{\sin 2 \varphi 2}{I_{2}^{2}}\right)\right] \text {, }
\end{aligned}
$$


where

$$
I_{j}=1+u^{4}-2 u^{2} \cos 2^{+} j(j=1,2) .
$$

It is easily verified that (0.73a), (8.74b) satisfy (2.13) and (8.73b), (8.74a) satisfy the second equation in (2.9).

Formulae $(5.55 \mathrm{a}, \mathrm{b})$ and $(5.56)$ for the moments at the centre reduce to

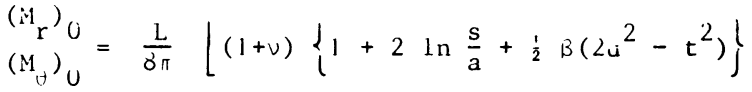

$$
\begin{aligned}
& \left.+(1-v)\left\{1+\frac{u^{2}}{2 k}\left(k^{2}+3-2 u^{2}\right)\right\} \cos 2 \gamma \cos 2 \theta\right\} \text {, } \\
& \left(M_{r y}\right)_{0}=\frac{(1-v) L}{8 \pi}\left[1+\frac{u^{2}}{2 k}\left(,^{2}+3-2 u^{2}\right)\right] \cos 2 \gamma \sin 2 \theta .
\end{aligned}
$$

For $\gamma=\pi / 4$ it is checked that the formulae $(8.73)-(8.76)$ are in agreement with those obtained by applying equations (2.44)-(2.46) of [3], which were derived by a different nethod.

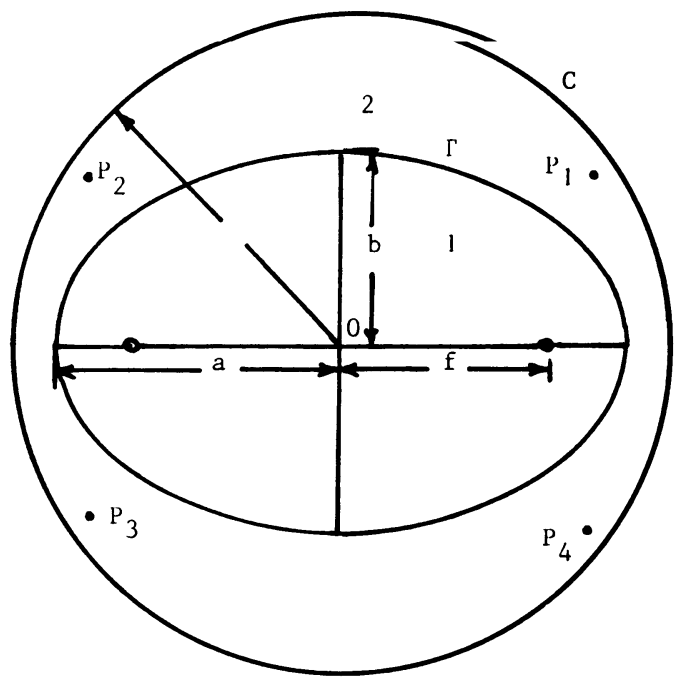

FIG. 1

ACKNOWLEDGEMENT. The author is indebted to Mrs. Eman S. Al-Khammash who typed the manuscript

\section{REFERENCES}

I. NADAI, $\Lambda$., Die Verbiegungen in einzelnen Punkten understützen Kriesförmiger Platten, Z. Phys.23, 366-376 (1922).

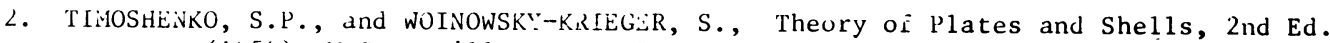
(1954), NicCraw-Hill, licw York.

3. BASSALI, W.A., The Transverse Flexure of Thin Elastic plates Supported at Several Points, proc. Cauis. Phil. Soc. 53, 723-743 (1957). 
4. BASSALI, W.A., Problems Concerning the Benuing of Isotropic Thin Elastic Plates Subject to Various Distributions of vornal Pressures, Proc. Camb. Phil. Soc. 24, 205-287 (1958).

3. YU, J.C.L., and PAN, H.H., Uniformly Loaued Circular Plate Supported at Discrete Points, Intern. J. liech. Sci. 3, 333-40 (1966).

6. LEISSA, A.W., and WELLS, L.T., Bending of a Uniformly Loaded Circular Plate on Interior Point Supports, Fifth U.S. Nat. Congr. Appl. Mech., 295 (June 1966).

7. KIKSTEIN, A.F., PLLL, W.H., WOOLLEY, R.M. and DAVIS, L.J., Deflection of Central1y Loaded Thin Circular Elastic Plates on Equally Spaced Point Supports, Engineering and Instrumentation, Sec. C, J. Research, Nat. Bur. St dnd. 70C, 4, 227-244 (1966).

3. KIRSTELN, A.F., and WOOLEEY, R.M., Symmetrical Benuing of Thin Circular Elastic Plates on Equally Spaced Point Supports, Engineering and Instrumentation, Sec. C, J. Research, Nat. Bur. Stand. 71C, 1, 1-10 (1967).

y. KISTEIN, A.F., and WOOLLEY, R.M., Deflection of Thin Circular Elastic Plates Under Symmetrically Distributed Loading, Engineering and Instrumentation, Sec. C, J. Researcị, Nat. Bur. Stand. 72C, I, 21-26 (1968).

10. Valihan, H., Deflection of Uniformly Loaded Circular Plates Upon Equispaced Point Supports, J. Str. Aila1. 5, 2, 115-120 (1970).

11. ChA.VTARAMUNGKORN, K., KARASUDHI, P. and LEE, S.L., Eccentrically Loaded Circular Plates Oi Columns, J. Struct. Div. Proc. ASCE 99 STI, 234-240 (1973).

12. WILlIAMS, R., and BRINSON, H.F., Circular Plates on Multipoint Supports, J. Frank. Instit. 297, 6, 429-447 (1974).

13. BASSALI, W.A. and NASSIF, M., A Thin Circular Plate Normally and Uniformly Loaded Over a Cuncentric Elliptic Patch, Proc. Cant. Phil. Soc. 55, 101-109 (195y).

14. BASSALI, W.A., Bending of a Thin Circular Plate Under Hydrostatic Pressure Over a Concentric Ellipse, Proc. Camb. Phil. Soc. 55, 110-120 (1959).

15. BASSALI, W.A., The Transverse Flexure of a Thin Circular Plate Subject to Parabolic Loading Over a Concentric Ellipse, J. Appl. Math. Phys. (ZANP) 11, 176-191 (1960).

16. FRISCHBIER, R., and LUCHT, W., The Circular Plate Subject to Constant Polygonal Load, Zeit. Angew. Math. Mech. (ZAMM) 50, 10, 593-605 (1970). 


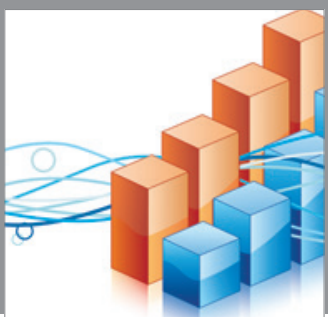

Advances in

Operations Research

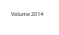

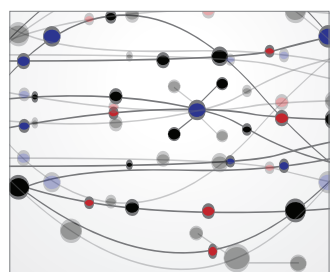

\section{The Scientific} World Journal
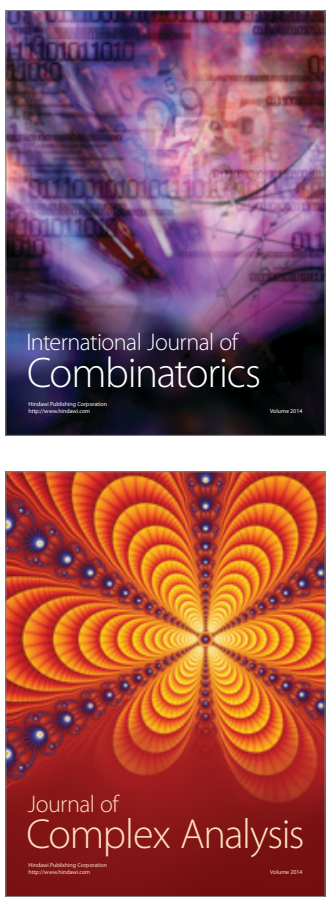

International Journal of

Mathematics and

Mathematical

Sciences
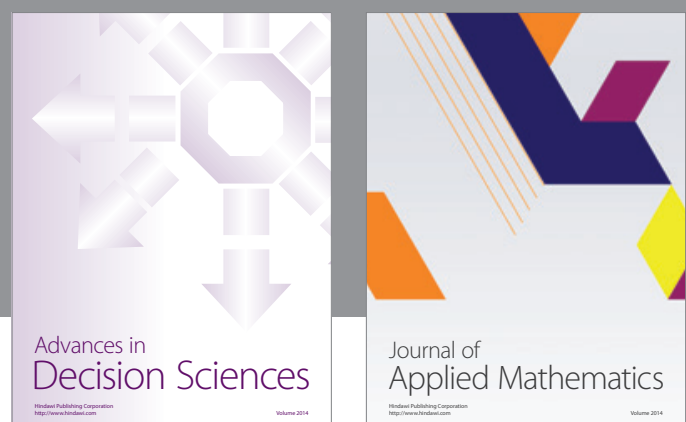

Journal of

Applied Mathematics
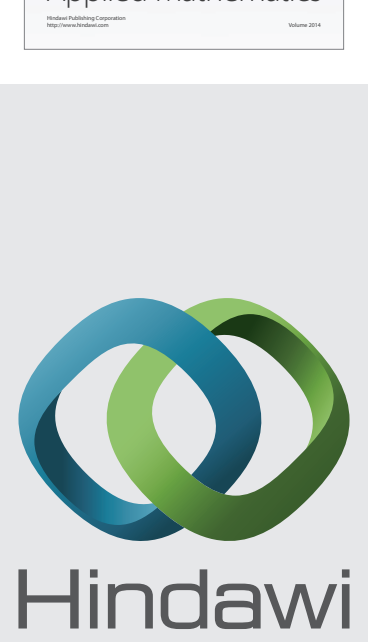

Submit your manuscripts at http://www.hindawi.com
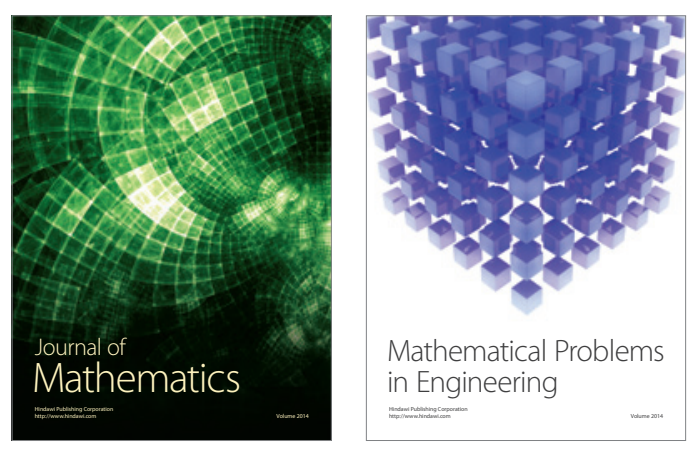

Mathematical Problems in Engineering
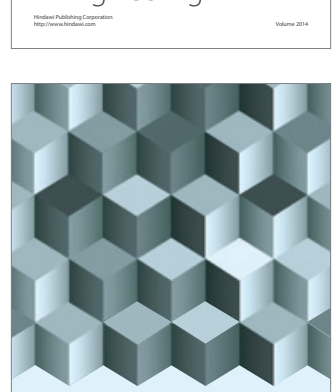

Journal of

Function Spaces
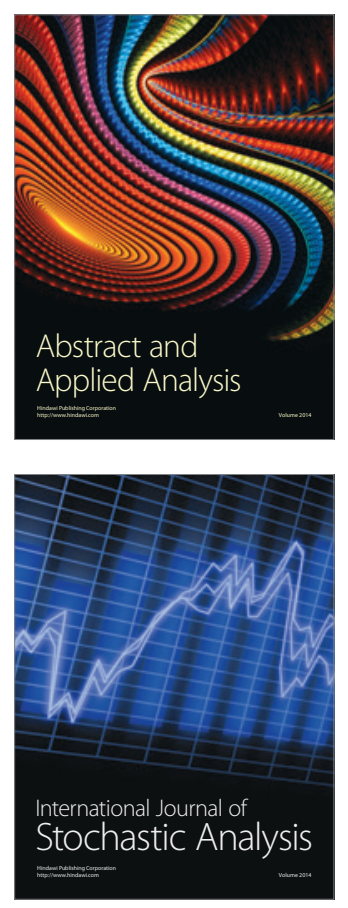

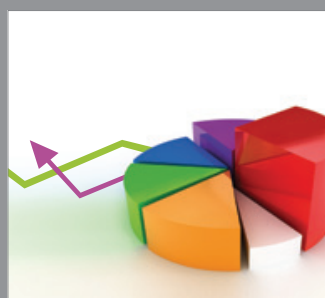

ournal of

Probability and Statistics

Promensencen
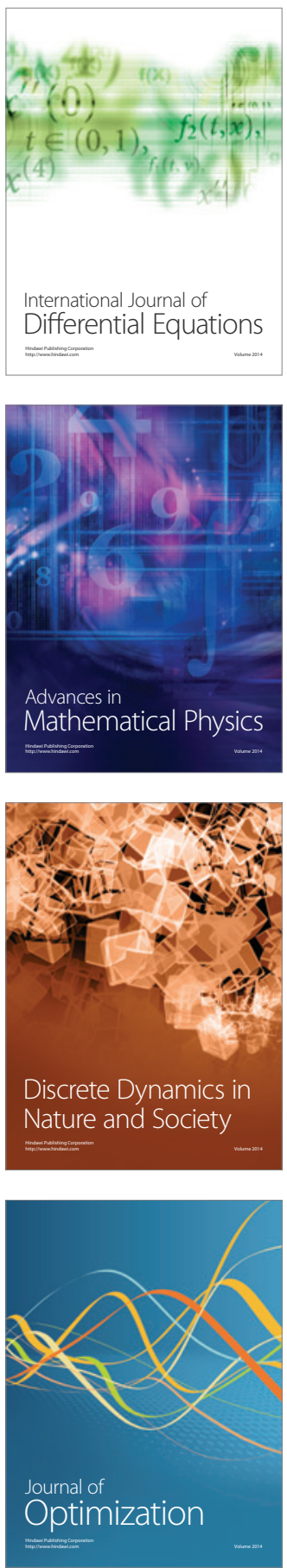